\title{
Energy Eigenvalue Level Motion with Two Parameters
}

\author{
Willi-Hans Steeb, Yorick Hardy, and Ruedi Stoop \\ International School for Scientific Computing, Rand Afrikaans University, \\ P.O. Box 524, Auckland Park 2006, South Africa
}

Reprint requests to Prof. W.-H. Steeb; E-mail: whs@na.rau.ac.za

Z. Naturforsch. 56 a, 565-567 (2001); received April 17, 2001

From the eigenvalue equation $\hat{H}_{\lambda}\left|\psi_{n}(\lambda)\right\rangle=E_{n}(\lambda)\left|\psi_{n}(\lambda)\right\rangle$ where $\hat{H}_{\lambda}=\hat{H}_{0}+\lambda \hat{V}$ one can derive an autonomous system of first order ordinary differential equations for the eigenvalues $E_{n}(\lambda)$ and the matrix elements $V_{m n}(\lambda):=\left\langle\psi_{m}(\lambda)|\hat{V}| \psi_{n}(\lambda)\right\rangle$ where $\lambda$ is the independent variable. We derive the partial differential equations for the extended case $\hat{H}_{\lambda_{1}, \lambda_{2}}=\hat{H}_{0}+\lambda_{1} \hat{V}_{1}+\lambda_{2} \hat{V}_{2}$, where $\lambda_{1}$ and $\lambda_{2}$ are the independent variables. Some applications of this system of partial differential equations are discussed.

Key words: Eigenvalue Level Motion; Partial Differential Equations.

Several authors [1 - 7] showed that starting from the eigenvalue equation $\hat{H}_{\lambda}\left|\psi_{n}(\lambda)\right\rangle=E_{n}(\lambda)\left|\psi_{n}(\lambda)\right\rangle$ with $\hat{H}_{\lambda} \equiv \hat{H}_{0}+\lambda \hat{V}$ one can derive an autonomous system of first order differential equations for the eigenvalues $E_{n}(\lambda)$ and the matrix elements $V_{m n}(\lambda)$, where $\lambda$ is the independent variable. To solve the dynamical system we need the initial values $E_{n}(\lambda=0)$ and $\left|\psi_{n}(\lambda=0)\right\rangle$. Thus one finds the "motion" of the energy levels $E_{n}(\lambda)$. The evolution of the eigenvectors $\left|\psi_{n}(\lambda)\right\rangle$ can also be derived. The assumptions are that the Hamilton operator has a discrete spectrum and that the eigenvalues are not degenerate. Furthermore, the eigenfunctions are real orthogonal and form a basis in the underlying Hilbert space. It is also assumed that the expansion does not diverge. The eigenvalues and eigenfunctions of the operator $\hat{H}_{0}$ are known. Degeneracies of eigenvalues are in general related to symmetries of the Hamilton operator. If the Hamilton operator admits discrete symmetries the Hilbert space can be decomposed into invariant subspaces. These invariant subspaces are again Hilbert spaces, and the system of differential equations can be applied to these subspaces.

In many systems we have two or more external parameters. We consider the case with two external parameters. We derive the partial differential equations for this system. The assumptions given above also apply for this case. Thus the Hamilton operator is of the form

$$
\hat{H}_{\lambda_{1}, \lambda_{2}}=\hat{H}_{0}+\lambda_{1} \hat{V}_{1}+\lambda_{2} \hat{V}_{2} .
$$

Let $\lambda=\left(\lambda_{1}, \lambda_{2}\right)$. We define

$$
p_{k n}(\lambda):=\left\langle\psi_{n}(\lambda)\left|\hat{V}_{k}\right| \psi_{n}(\lambda)\right\rangle
$$

and

$$
V_{k m n}(\lambda):=\left\langle\psi_{m}(\lambda)\left|\hat{V}_{k}\right| \psi_{n}(\lambda)\right\rangle, m \neq n,
$$

where $k=1,2$. We use the orthogonality relation

$$
\left\langle\psi_{m}(\lambda) \mid \psi_{n}(\lambda)\right\rangle=\delta_{m n}
$$

and the completeness relation

$$
1=\sum_{n \in I}\left|\psi_{n}(\lambda)\right\rangle\left\langle\psi_{n}(\lambda)\right| .
$$

From the orthogonality relation we find

$$
\left\langle\frac{\partial\left\langle\psi_{m}(\lambda)\right|}{\partial \lambda_{k}} \mid \psi_{n}(\lambda)\right\rangle+\left\langle\psi_{m}(\lambda)\right| \frac{\partial\left|\psi_{n}(\lambda)\right\rangle}{\partial \lambda_{k}}=0 .
$$

Using these properties, we obtain the following system of partial differential equations

$$
\begin{gathered}
\frac{\partial E_{n}}{\partial \lambda_{k}}=p_{k n}, \frac{\partial p_{k n}}{\partial \lambda_{j}}=\sum_{m(\neq n)} \frac{V_{j n m} V_{k m n}+V_{k n m} V_{j m n}}{E_{n}-E_{m}} \\
\frac{\partial V_{k m n}}{\partial \lambda_{j}}=\sum_{l(\neq m, n)}\left(\frac{V_{j m l} V_{k l n}}{E_{m}-E_{l}}+\frac{V_{j l n} V_{k m l}}{E_{n}-E_{l}}\right) \\
+\frac{V_{j m n}\left(p_{k m}-p_{k n}\right)}{E_{n}-E_{m}}
\end{gathered}
$$


where $k=1,2$ and $j=1,2$. This system of partial differential equations has to be solved with the initial conditions

$$
\begin{aligned}
& E_{n}\left(\lambda_{1}=0, \lambda_{2}=0\right), p_{k n}\left(\lambda_{1}=0, \lambda_{2}=0\right), \\
& V_{k m n}\left(\lambda_{1}=0, \lambda_{2}=0\right) .
\end{aligned}
$$

Thus the eigenvalues and eigenvectors of $\hat{H}_{0}$ must be known. Using the eigenvectors, we calculate $p_{k n}$ $\left(\lambda_{1}=0, \lambda_{2}=0\right)$ and $V_{k m n}\left(\lambda_{1}=0, \lambda_{2}=0\right)$. As mentioned above, we have to do group theoretical reduction to the invariant subspaces and then apply the equations to the subspaces.

The extension to $n$ external parameters is straightforward. For two and more external parameters we must take into account the theorem of Wigner and von Neumann [8] that was discovered in the very earliest days of quantum mechanics almost 70 years ago. The theorem says that, among all self-adjoint matrices, those with a degenerate eigenvalue have codimension three. When there is time-reversal invariance, as in the present case, one should be looking at real symmetric matrices, in which case the corresponding theorem says that they have co-dimension two. This is usually interpreted as saying that it requires at least two external parameters before one can expect any kind of eigenvalue crossing, unless there is some symmetry reason that prevents the crossings being avoided, as the parameter is varied. This fact must be taken into account when we solve the system of partial differential equations given above.

An analytic solution can be found with the help of the ansatz (power series)

$$
\begin{aligned}
& E_{n}(\lambda)=\sum_{i, j=0}^{\infty} E_{i j}^{n} \lambda_{1}^{i} \lambda_{2}^{j}, \\
& p_{k n}(\lambda)=\sum_{i, j=0}^{\infty} p_{i j}^{k n} \lambda_{1}^{i} \lambda_{2}^{j} .
\end{aligned}
$$

We find in second order of approximation

$$
\begin{aligned}
E_{n}\left(\lambda_{1}, \lambda_{2}\right) \approx & E_{n}(0,0)+E_{10}^{n} \lambda_{1}+E_{01}^{n} \lambda_{2}+E_{20}^{n} \lambda_{1}^{2} \\
& +E_{11}^{n} \lambda_{1} \lambda_{2}+E_{02}^{n} \lambda_{2}^{2},
\end{aligned}
$$

where

$$
E_{10}^{n}=p_{1 n}(0,0), E_{01}^{n}=p_{2 n}(0,0)
$$

and

$$
\begin{aligned}
& E_{20}^{n}=\sum_{m(\neq n)} \frac{V_{1 m n}(0,0) V_{1 m n}(0,0)}{E_{n}(0,0)-E_{m}(0,0)}, \\
& E_{02}^{n}=\sum_{m(\neq n)} \frac{V_{2 m n}(0,0) V_{2 m n}(0,0)}{E_{n}(0,0)-E_{m}(0,0)}, \\
& E_{11}^{n}=\sum_{m(\neq n)} \frac{2 V_{1 m n}(0,0) V_{2 m n}(0,0)}{E_{n}(0,0)-E_{m}(0,0)} .
\end{aligned}
$$

Let us give some examples where we can apply this system of differential equations. The Hubbard model is given by

$$
\hat{H}=t \sum_{i, j} \sum_{\sigma} c_{i \sigma}^{\dagger} c_{j \sigma}+U \sum_{i} n_{i \uparrow} n_{i \downarrow}
$$

with the two parameters $t$ and $U$, where $i, j$ are lattice points and $c_{i \sigma}^{\dagger}$ and $c_{i \sigma}$ are Fermi creation and annihilation operators with spin $\sigma$. We could scale $\hat{H}$ with respect to $t$ or $U$. This would lead to the case with one parameter. If we would consider the case with an external magnetic field

$$
\begin{aligned}
\hat{H}= & t \sum_{i, j} \sum_{\sigma} c_{i \sigma}^{\dagger} c_{j \sigma}+U \sum_{i} n_{i \uparrow} n_{i \downarrow} \\
& +\frac{1}{2} \eta \sum_{i}\left(c_{i \uparrow}^{\dagger} c_{i \uparrow}-c_{i \downarrow}^{\dagger} c_{i \downarrow}\right),
\end{aligned}
$$

we would have two parameters again. Another example is the spin system

$$
\hat{H}=a \sum_{j} \sigma_{3 j} \sigma_{3 j+1}+b \sum_{j} \sigma_{1 j} .
$$

Finally, systems with an external electric and magnetic field could be studied with this system of partial differential equations, for example [7]

$$
\hat{H}=-\frac{\hbar^{2}}{2 \theta} \frac{\mathrm{d}^{2}}{\mathrm{~d} \phi^{2}}+i \mu \mathcal{H} \frac{\mathrm{d}}{\mathrm{d} \phi}-p \mathcal{E} \cos (\phi) .
$$


[1] P. Pechukas , Phys. Rev. Lett. 51, 943 (1983).

[2] T. Yukawa, Phys. Lett. A116, 227 (1985).

[3] K. Nakamura and M. Lakshmanan, Phys. Rev. Lett. 57, 1661 (1986).

[4] W.-H. Steeb, A. J. van Tonder, C. M. Villet, and S. J. M. Brits, Found. Phys. Lett. 1, 147 (1988).

[5] W.-H. Steeb, J. Phys. A: Math. Gen. 21, 2657 (1988).
[6] W.-H. Steeb, J. Phys. Soc. Japan 57, 1930 (1988).

[7] W.-H. Steeb, Hilbert Spaces, Wavelets, Generalised Functions and Modern Quantum Mechanics, Kluwer Academic Publishers, Dordrecht 1998.

[8] J. von Neumann and E. Wigner, Phys. Z. 30, 467 (1929). 